\title{
Computer-aided Diagnosis of Glaucoma Using Fundus Images
}

\author{
Yongli, $\mathrm{Xu}$ \\ Department of Mathematics, \\ Beijing University of Chemical Technology, \\ Beijing, China \\ e-mail: xuyongli2312@sina.com \\ Xin, Jia \\ Department of Mathematics, \\ Beijing University of Chemical Technology, \\ Beijing, China \\ e-mail: 786728424@qq.com
}

\author{
Man, Hu, Department of Ophthalmology, Beijing \\ Children's Hospital, Capital Medical University, \\ Beijing, China, \\ e-mail: human1125@163.com
}

\begin{abstract}
Glaucoma is a chronic eye disease which cannot be cured, so that detecting the disease in time is important. Machine learning for glaucoma diagnosis has achieved great development in recent years. In this paper, we present an algorithm for glaucoma diagnosis from optic disc and optic cup boundary lines in fundus images based on doctors' knowledge. We do meticulous division, scaling transformation and principal component analysis on the optic disc and optic cup boundary lines to extract features. The extracted features correspond well with doctors' knowledge. Therefore, we can make an intuitive explanation for the diagnosis results to doctors, rather than just as a black-box prediction. On a real sample set, the proposed feature extraction and diagnosis algorithms achieve high prediction accuracy.
\end{abstract}

Keywords- machine learnin; glaucoma diagnosis; computer-aided diagnosis; fundus images; ISNT rule.

\section{INTRODUCTION}

Glaucoma is a group of eye diseases that have common traits such as, high eye pressure, damage to the optic nerve head $(\mathrm{ONH})$ and gradual vision loss. Glaucoma is the second leading cause of blindness with a mean prevalence of $2.4 \%$ for all ages [1]. It affects the peripheral vision if left untreated and over time it leads to tunnel vision or complete vision loss. The damage is irreversible and treatment can only prevent or reduce further damage. Therefore, early diagnosis and treatment is essential for patients to preserve their vision [2]. The assessment of the $\mathrm{ONH}$ is important in glaucoma diagnosis. Fundus camera is the most commonly used glaucoma screening equipment. From fundus images, a large number of image features of the $\mathrm{ONH}$ can be extracted. Among them, two major ones used for glaucoma diagnosis are optic disc (OD) and optic cup. The optic disc is the visible portion of the optic nerve, from which the nerve fibres exit the eye [3]. The central depression of the OD is known as the optic cup and the

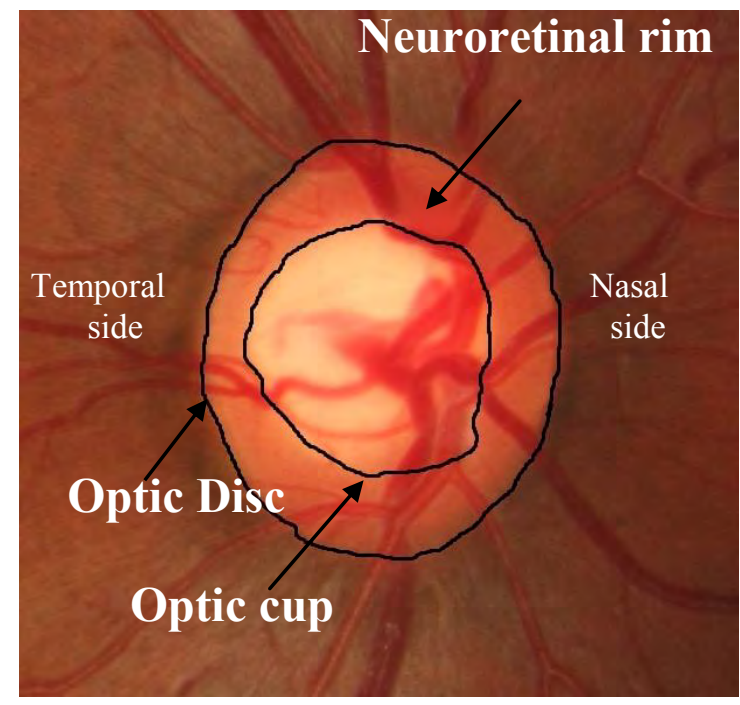

Figure 1. The main structures of the $\mathrm{ONH}$.

area around the optic cup is known as the neuroretinal rim. The main structures of the ONH are shown in Fig. 1.

The boundary lines of the optic disc and optic cup are important in identifying glaucoma. The cup-to-disc ratio (CDR) and ISNT rule are two key indexes to distinguish glaucoma from normal eyes. The CDR is defined as the ratio of the vertical cup diameter to the vertical disc diameter [4]. Glaucoma leads to structural changes of the neuroretinal rim: it gets thinner while the cup is expanding. Generally, the CDR of glaucoma is larger than normal eyes. ISNT rule is another important index to distinguish between normal and glaucomatous eyes: normal optic discs usually meet a rule that the inferior neuroretinal rim is the widest portion of the rim, followed by the superior rim, the nasal rim and the temporal rim [5].

Due to the variety and complexity of the disease pathology, the diagnosis of glaucoma relies heavily on the experiences of doctors. Glaucoma's irreversibility and shortage of glaucoma specialist demand for an economic, 
effective and automatic glaucoma screening system. Computer-aided diagnosis for glaucoma has achieved great development in recent years. A number of machine learning algorithms, such as artificial neural networks (ANNs), support vector machines (SVMs), decision tree, linear discriminant analysis (LDA), etc, have been used for glaucoma diagnosis with visual field, OCT and HRT [6-9]. In resent years, many glaucoma diagnosis learning algorithms from fundus images were proposed. The use of priori knowledge is an important way to improve the prediction accuracy of learning algorithms [10]. However, among these works, ISNT rule has not been directly utilized.

Bock et al. (2010) used principal component analysis (PCA), Fourier analysis and spline interpolation methods to extract features from fundus images and then used SVM for glaucoma classification [11]. Liu et al. (2013) divided optic disc into grids, calculated a histogram of the green channel and some other modes, resulting in a high dimensional vector for each grid. The final medical image features are obtained as the standard derivation of the vectors for all grids [12]. These two methods did not use doctors' priori knowledge about the optic disc and cup, but dealt with fundus images as general photographs.

Nayak et al. (2009) extracted features such as cup to disc area ratio, ratio of the distance between optic disc center and optic nerve head to diameter of the optic disc [13]. These features are used for classifying the normal and glaucoma images with neural network classifier. Chen et al. (2013) presented a superpixel classification based disc and cup segmentations for glaucoma screening [14]. They computed centre surround statistics from superpixels and unified them with histograms for disc and cup segmentation. Based on the segmented disc and cup, vertical cup to disc ratio is computed for glaucoma screening. Zhang et al. (2011) exacted features from optic disc and cup boundary lines, such as vertical cup-disc ratio, rim inferior (superior, nasal, temporal) thickness, area of optic cup and disc [15]. They selected the most valuable features from image features and medical screening. And then LDA, SVM and AdaBoost classifiers were built using the selected features. These three methods utilized doctors' prior knowledge about the optic disc and cup, but did not used ISNT rule directly.

In this paper, we present an algorithm for feature extraction from optic disc and optic cup boundary lines, based on which glaucoma diagnosis prediction algorithms are designed. We do meticulous partition for the optic disc and optic cup, in order to catch complete information of the optic disc and optic cup boundary lines. Next, we do scaling transformation for cup-to-disc radius radio in each small area to highlight differences between glaucoma and normal eyes on ISNT rule. Finally, PCA method is used to select the principal components of sample set, and the inner products of each sample and these principal components are selected as new features of the sample. These features include ones which are concerned about by doctors (average cup-to-disc radius radio and ISNT rules). The proposed algorithm has both high accuracy and interpretability. The basic principle of the algorithm is consistent with doctors' diagnosis thinking. Consequently the algorithms are easy to understand by doctors.

\section{METHODS}

This paper proposes a model for glaucoma diagnosis which can incorporate machine learning methods and doctors' prior knowledge. In medical diagnosis problems, there are both medical records of patients and doctors' prior knowledge. We combine these two kinds of information to design a model. Medical records include patients' physiological characteristics and medical examination results. By preprocessing, the information of each patient is reorganized into a sample. Every sample is composed of feature vector $\mathrm{X}$ and label $\mathrm{Y}$. In this paper, $\mathrm{X}$ represents optic disc and optic cup boundary lines and $\mathrm{Y}$ represents the normal eye or glaucoma $(+1$ stands for glaucoma and -1 stands for normal eye). Doctors' knowledge comes from glaucoma specialists' experience and clinical thinking based on the experience. The experience is usually vague, emotional and is not described quantitatively. We refine the doctors' knowledge into rules to guide learning algorithm design.

The learning algorithm includes two parts: feature extraction and classification. The feature extraction is the base of classification and classification gives a diagnostic criteria. Finally, the learning algorithm obtains two aspects of result. The first is the classification result: diagnosis for new patients according to their fundus photographs. The second is the interpretation of extracted features, which corresponds to rules from doctors' knowledge.

In this paper, support vector machine (SVM) is used for classification. SVM was introduced by Vapnik [16] in 1995 and since then became a popular method in pattern recognition. SVM can efficiently perform a non-linear classification using what is called the kernel trick, implicitly mapping their inputs into high-dimensional feature spaces [17]. SVM has been widely used in computer-aided diagnosis [18, 19]. In this study, we use SVM to classify glaucoma and normal eyes. In the following, we focus on feature extraction.

\section{A. Motivation}

(1) How to quantify ISNT rule?

In the existing machine learning algorithms for glaucoma diagnosis, the ISNT rule is not been reflected directly. The reason is that, the ISNT rule is a qualitative rule, but not quantitatively characterized. That is, given optic disc and cup boundary lines of two fundus photographs, there is not a specific criteria to determine one is more consistent with the ISNT rule than another. Therefore, we want to give a quantitative indicator to reflect the ISNT rule. On the one hand, it will draw lessons from doctor's diagnosis thinking to improve prediction accuracy. On the other hand, diagnosis results based on this indicator are more likely to be understood and accepted by doctors. A simple indicator can be defined as follows: 


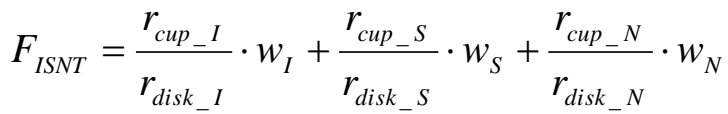

$$
\begin{aligned}
& +\frac{r_{\text {cup_t } T}}{r_{\text {disk_t }}} \cdot w_{T}
\end{aligned}
$$

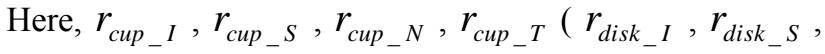
$r_{\text {disk_N }}, r_{\text {disk_T }}$ ) denote the optic cup (disc) radius of inferior, superior, nasal and the temporal areas. And $w_{I}, w_{S}, w_{N}, w_{T}$ denote the weights of cup-to-disk radius ratio of inferior, superior, nasal and temporal areas.

Let $w_{I}, w_{S}, w_{N}, w_{T}$ be four descending real numbers (e.g.: 4,3,2,1). Then, the $F_{I S N T}$ score of normal eyes tend to smaller than that of glaucoma. There are two reasons: On the one hand, cup-to-disc radius ratios in all the four areas of normal eyes tend to smaller than that of glaucoma. On the other hand, for normal eyes, areas with smaller cup-to-disc radius ratios (inferior and superior areas) correspond to larger weights and areas with larger cup-todisc radius ratios (nasal and temporal areas) correspond to smaller weights. For example, consider a general normal eye, a normal eye with physiologic large cup and a glaucoma with cup-to-disc radius ratio of inferior, superior, nasal and the temporal areas are $(0.3,0.4,0.6,0.7)$, $(0.4,0.5,0.7,0.8)$ and $(0.6,0.6,0.6,0.6)$ respectively. Then, the $F_{I S N T}$ score of the above three eye is $4.3,5.3$ and 6 respectively, in the case $w_{I}=4, w_{S}=3, w_{N}=2, w_{T}=1$. Therefore, we can expect the $F_{I S N T}$ score is an effective feature to distinguish glaucoma and normal eyes.

However, in the formula (1), there are two aspects of information need to be given in advance: Which direction in the 360-degree should be selected as inferior, superior, nasal and temporal directions? How to decide the specific values of $w_{I}, w_{S}, w_{N}$ and $w_{T}$ ? We hope that the two aspects of information can be given based on the sample set.

(2) Use cup-to-disc radius radio or area radio?

In existing machine learning methods for glaucoma diagnosis, some use cup-to-disc radius radio as a feature (such as [14]), and some use cup-to-disc area radio (such as [15]). In clinical diagnosis, doctors do not have a uniform standard to use which one. Therefore, we want to give a more efficient scale of cup-to-disc ratio based on sample set to distinguish between glaucoma and normal eyes. The target is that the $F_{I S N T}$ score of glaucoma and normal eye can have a greater difference under the selected cup-to-disc ratio scale. The general form of scale we give is $\left(\frac{r_{\text {cup }}}{r_{\text {disk }}}\right)^{q}$, for some $\mathrm{q}>0$. Here, $\mathrm{q}$ need be selected based on sample set. In particular, when $\mathrm{q}=2$, we have $\frac{S_{\text {cup }}}{S_{\text {disk }}}=\left(\frac{r_{\text {cup }}}{r_{\text {disk }}}\right)^{q}$. Here, $S_{\text {cup }}$ and $S_{\text {disk }}$ respectively represent the area of the optic cup and optic disc. Therefore, if we consider the scale choice above, indicator $F_{I S N T}$ score in formula (1) can be generalized as follows:

$$
\begin{aligned}
F_{I S N T_{-} q}= & \left(\frac{r_{\text {cup_I }} I}{r_{\text {disk_I }}}\right)^{q} \cdot w_{I}+\left(\frac{r_{\text {cup_s }} S}{r_{\text {disk_s }} S}\right)^{q} \cdot w_{S} \\
& +\left(\frac{r_{\text {cup_ }} N}{r_{\text {disk_N }}}\right)^{q} \cdot w_{N}+\left(\frac{r_{\text {cup_T }} T}{r_{\text {disk_t }}}\right)^{q} \cdot w_{T}
\end{aligned}
$$

\section{B. Feature extraction techniques}

Some rules according to doctors' knowledge are applied on the feature extraction part to exact features from optic disc and optic cup boundary lines, thereby improving the prediction accuracy and exploring the nature of the data structure. The rules used in this paper include:

(a) The optic discs and cups of both glaucoma and normal eye are approximate ellipses.

(b) The average cup-to-disc radius ratio of glaucoma is usually larger than that of normal eye.

(c) The neuroretinal rim of normal eyes usually meets the ISNT rule, while that of glaucoma do not meet the rule.

These rules are used to design methods for feature extraction, which is shown in Fig .2. The feature extraction methods in this paper base on the optic cup and optic disc boundary lines in fundus images. A large number of methods for optic disc and cup segmentation have appeared $[14,20]$. However, there is still large difference between optic cup boundary lines drawn by automatic algorithms and those drawn by glaucoma specialist [20]. The proposed feature extraction algorithm need base on more accurate optic cup boundary lines. Therefore, we use the optic disc and optic cup boundary lines drawn by doctors and mainly discuss how to extract features from these boundary lines.

(1) Segmentation for optic disc and optic cup

Considering the optic disc and optic cup are approximate ellipses, we divide them into 360 fan-shaped areas which have the same center with the optic cup. Then average cup-to-disc radius ratio $r^{i}{ }_{\text {cup }} / r^{i}{ }_{\text {disk }}$ is calculated in each area and a 360 dimensional vector is obtained.

\section{(2) $Q$-transform}

The difference of average cup-to-disc radius ratio between some early glaucoma and normal eyes with physiologic large cups is not obvious. To highlight the difference of glaucoma and normal eyes on the ISNT rule, we design a q-transform: multiply the cup-to-disc radius ratio by the q-th power in each of the 360 parts, for some $q>0$. The geometric explanation of q-transform is that, if we maintain optic disc radius, new optic cup radius shrinks $\quad(\mathrm{q}>1) \quad$ or $\quad$ expands $\quad(\mathrm{q}<1)$ to $\bar{r}_{\text {cup }}=r_{\text {disk }} \times\left(r_{\text {cup }} / r_{\text {disk }}\right)^{q}$, in each of the 360 parts. 
Methods

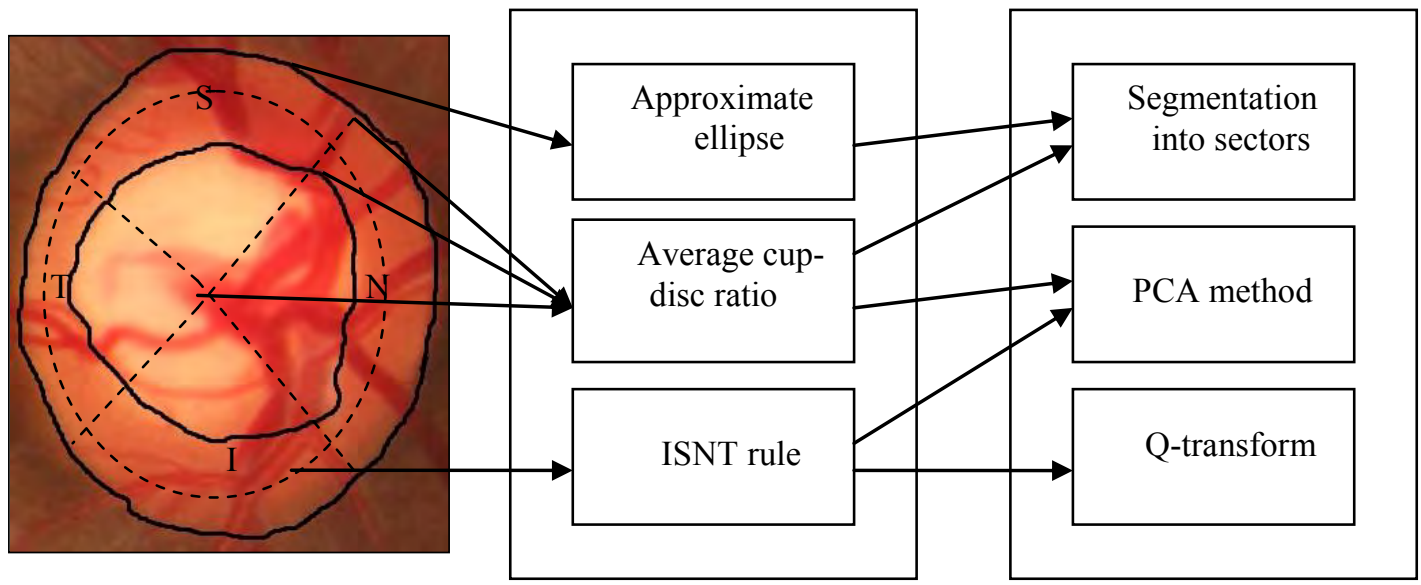

Figure 2. Diagram for feature extraction

Furthermore, when $\mathrm{q}>1(\mathrm{q}<1)$, the smaller the cup-disc ratio is, the greater the optic cup boundary shrinks (expands). Therefore, q-transform could highlight the asymmetry of the rim of physiologic large cups.

\section{(3) PCA method}

The 360-dimensional vector contains comprehensive information of the optic disc and optic cup boundary lines. However, if data dimension is high, satisfying classification results cannot be obtained. Therefore, we use PCA method to extract features from the sample set. PCA is a technology to analysis and simplify data. This method maintains features with large contribution to the variance and removes that with small contribution to reduce dimensionality [21]. Since the average cup-to-disc radius ratio and ISNT rule are important features of fundus image, we try to

use PCA to select principal components corresponding to them.

The input of each sample is a 360-dimensional vector $x=\left(x_{1}, x_{2}, \Lambda, x_{360}\right)^{\prime}$. We use PCA to obtain the first k principal components $u_{1}, \ldots, u_{k} \in R^{360}$. With these principal components, we can denote a sample $x$ as $a=\left[u_{1}^{\prime} \cdot x, u_{2}^{\prime} \cdot x, \Lambda, u_{k}^{\prime} \cdot x\right]^{\prime}$. From the above equation we can see that each component stands for the weights of the weighted average cup-to-disc radius ratio in 360 parts. For example, if a principal component is $\quad u=[1 / \sqrt{360}, 1 / \sqrt{360}, \Lambda, 1 / \sqrt{360}], \quad$ then $u^{\prime} \cdot x$ represents the average cup-to-disc radius ratio corresponding to $\mathrm{x}$; and if a principal component is $\quad v=\left[0, \Lambda, 1_{1}^{\prime} \sqrt{99}, 2_{I} 4^{1 / 4} 4^{90}, \Lambda, 0\right] \quad, \quad$ then

$v^{\prime} \cdot x_{i}$ represents the average cup-to-disc radius ratio in inferior area corresponding to $\mathrm{x}$. We expect PCA can extract weights which reflect the average cup-to-disc radius ratio and ISNT rule, based on samples. For the principal component corresponding to ISNT rule $u_{I S N T}=\left[w_{1}, w_{2}, \Lambda, w_{360}\right]^{\prime}$, the formula (2) can be generalized to the following form:

$$
F_{\text {ISNT_q }}=\sum_{i=1}^{360}\left(\frac{r_{\text {cup }}^{i}}{r^{i}{ }_{\text {disk }}}\right)^{q} \cdot w_{i}
$$

\section{Algorithms for feature exaction from optic disk and optic cup boundary lines}

We propose feature extraction algorithms based on optic cup and optic disc boundary lines as follows.

\section{Algorithm I:}

Step 1: Divide the optic disc and optic cup into 360 parts evenly and calculate the average cup-to-disc radius ratio in each part to get a 360-dimensional vector $x=\left(x_{1}, \ldots, x_{360}\right)$.

Step 2: Multiple each element $x_{i}$ of the vector by q-th power to get a new elements $x_{i}^{q}$ and form a new 360-dimensional vector $x^{q}=\left(x_{1}^{q}, \Lambda, x_{360}{ }^{q}\right)$.

Step 3: By PCA method, extract the first $\mathrm{k}$ principal components of the 360-dimensional vector set $\left\{u_{1}, \ldots, u_{k}\right\}$, and use the inner product of a sample and each of the first $\mathrm{k}$ principal components $a_{j}=u_{j}^{\prime} \cdot x^{q}$ to constitute a kdimensional vector $\left(a_{1}, \ldots, a_{k}\right)^{\prime}$.

The process of Algorithm I is shown in Fig .3. The goal of dividing the optic disc and cup into 360 parts evenly is to take full advantage of fundus image details. Qtransformation is used to transform the scale of cup-todisc radius ratio, which provides a more flexible choice for feature extraction. The value of $\mathrm{q}$ will be selected based on the specific sample set. The PCA method is used to select features, which have large contribution to the variance. Since in clinical diagnosis, the average cup-todisc radius ratio and ISNT rule are important features of fundus image, we can expect the PCA method can obtain appropriate weights corresponding to them. 


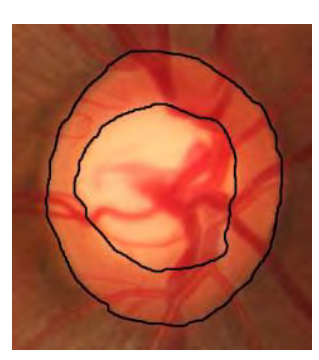

Cup and disc boundary lines

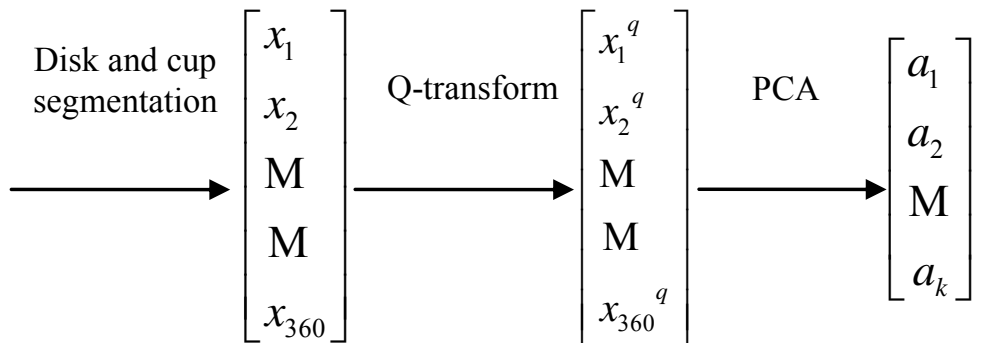

\section{Cup-disk} radius ratio
Projection to the first k principal components

Figure 3. Diagram of Algorithm I.

\section{RESULTS}

\section{A. Experiment design}

The performance of proposed algorithm is in investigated on a Retinal image data set obtained from Institute of Ophthalmology in Beijing Tongren Hospital. The sample set consists of 63 fundus images of normal eyes and 45 fundus images of glaucoma. The fundus images of glaucoma contain each 9 cases of level 1-5. Fundus images of normal eyes contain 45 common cases and each 6 cases of elliptical disk, physiologic large cup and cup off-center. The boundary lines of optic disc and optic cup in each fundus image are drawn by a doctor. We use Algorithm I to extract features of all images and then used SVM for classification.

Different parameters correspond to different feature extraction results. For data obtained by each feature extraction method, we do 1000 simulations. Every time, 108 samples are randomly divided into a training set and a test set, which contains 72 and 36 samples respectively. The training set is used to obtain the prediction function and test set is used for final test. The prediction accuracy is defined to be the ratio of the number of samples correctly predicted to the total number of samples in the test set. The mean prediction accuracy is defined as the prediction accuracy averaged over the 1000 simulations. We use the mean prediction accuracy to measure the prediction performance of the feature extraction and classification algorithms.

\section{B. Experiment results}

Table 1 shows the mean prediction accuracy of SVM and feature extraction Algorithm I (with different parameters $\mathrm{q}$ and k). In Table 1, "No PCA" represents mean prediction accuracy of SVM on original 360-dimension inputs without feature extraction by PCA, which is a reference for Algorithm I. From Table 1 we see that, for a fixed q, with the increase in the number of principal components, the mean prediction accuracy increases first and then decreases approximately. For any fixed q, the prediction accuracy of SVM based data with optimal number of principal components is much better than that without PCA and that with all 104 principal components. In all the stated cases for $\mathrm{q}$ and $\mathrm{k}$, when $\mathrm{q}=0.75$ and $\mathrm{k}=3$, the optimal prediction accuracy of $95.8 \%$ is obtained, which is much better than that of $85.3 \%$ without feature extraction (" $\mathrm{q}=1$ " and "no PCA").

Then, we compare prediction accuracy of SVM and Algorithm I with other feature extraction methods. Table 2 shows the mean prediction accuracy of SVM and some feature extraction methods. In Table 2, Average r-radio denotes average cup-to-disc radius radio in the range of 360 degrees; ISNT r-radio (a-radio) denotes average cupto-disc radius (area) radio in inferior, superior, nasal and temporal areas. From Table 2 we see that, compared to other methods, Algorithm I obtains the optimal mean prediction accuracy.

Table I: Mean prediction accuracy (\%) of SVM and feature extraction Algorithm I with different parameters $\mathrm{k}$

\begin{tabular}{|c|c|c|c|c|c|c|}
\hline \multicolumn{1}{|c|}{ q } & 0.25 & 0.5 & 0.75 & 1 & 2 & 3 \\
\hline $\begin{array}{l}\text { No } \\
\text { PCA }\end{array}$ & 81.3 & 83.8 & 84.6 & 85.3 & 87.5 & 88.4 \\
\hline 1 PC & 76.2 & 76.4 & 74.7 & 77.6 & 76.2 & 67.3 \\
\hline 1-2 PC & $\mathbf{9 3 . 8}$ & 93.1 & 91.5 & 91.2 & 83.6 & 75.2 \\
\hline $1-3$ PC & 91.2 & $\mathbf{9 5 . 2}$ & $\mathbf{9 5 . 8}$ & 86.4 & 82.7 & 81.9 \\
\hline 1-4 PC & 89.7 & 93.9 & 92.4 & $\mathbf{9 3 . 7}$ & $\mathbf{9 4 . 5}$ & 86.4 \\
\hline $1-5$ PC & 88.1 & 89.6 & 88.3 & 92.9 & 92.3 & $\mathbf{9 2 . 7}$ \\
\hline 1-6 PC & 86.9 & 88.2 & 87.6 & 91.2 & 90.9 & 89.8 \\
\hline All PC & 82.6 & 86.8 & 86.9 & 88.7 & 89.6 & 88.6 \\
\hline
\end{tabular}

Table II: Mean prediction accuracy (\%) of SVM and different feature extraction methods

\begin{tabular}{|c|c|c|c|c|}
\hline Method & $\begin{array}{c}\text { Average } \\
\text { r-radio }\end{array}$ & $\begin{array}{c}\text { ISNT } \\
\text { r-radio }\end{array}$ & $\begin{array}{c}\text { ISNT } \\
\text { a-radio }\end{array}$ & $\begin{array}{c}\text { Proposed } \\
\text { Method }\end{array}$ \\
\hline $\begin{array}{c}\text { Prediction } \\
\text { accuracy }\end{array}$ & 75.3 & 90.2 & 91.8 & $\mathbf{9 5 . 6}$ \\
\hline
\end{tabular}

\section{ACKNOWLEDGMENT}

This work was supported in part by the National Science Foundation of China, under Grants 11101024 and 11301021. 


\section{REFERENCES}

[1] B. Klein, R. Klein, W. Sponsel, et al., Prevalence of glaucoma: the beaver dam eye study, Ophthalmology, Vol. 99, pp. 1499-504, 1992.

[2] G. Michelson, S. Wärntges, J. Hornegger, B. Lausen, , The papilla as screening parameter for early diagnosis of glaucoma, Dtsch. Arztebl. Int. Vol. 105, pp. 583-589, 2008.

[3] P. N. Schacknow, J. R. Samples, The Glaucoma Book (First Edition). Portland, USA: Springer Publication, 2010

[4] J. Jonas, W. Budde, S. Panda-Jonas, Ophthalmoscopic evaluation of the optic nerve head, Survey of Ophthalmology, Vol. 43, pp. 293 $320,1999$.

[5] N. Harizman, C. Oliveira, A. Chiang, et al., The ISNT rule and differentiation of normal from glaucomatous eyes, Archives of ophthalmology, Vol. 124, pp. 1579-1583, 2006.

[6] K. Chan, T. W. Lee, P. A. Sample, et al. Comparison of machine learning and traditional classifiers in glaucoma diagnosis, IEEE Transactions on Biomedical Engineering, Vol. 49, pp. 963-974. 2002.

[7] L. M. Zangwill, K. Chan, C. Bowd, et al. Heidelberg retina tomograph measurements of the optic disc and parapapillary retina for detecting glaucoma analyzed by machine learning classifiers, Investigative ophthalmology and visual science, Vol. 45, pp. 3144 3151, 2004.

[8] D. Bizios, A. Heijl, J. L. Hougaard, et al. Machine learning classifiers for glaucoma diagnosis based on classification of retinal nerve fibre layer thickness parameters measured by Stratus OCT, Acta Ophthalmologica, Vol. 88, pp. 44-52, 2010.

[9] R. Koprowski, M. Rzendkowski and Z. Wróbel, Automatic method of analysis of OCT images in assessing the severity degree of glaucoma and the visual field loss, Biomedical engineering online, Vol. 13, No. 16, 2014.

[10] G. A. Drastal, C. A. Kulikowski, Knowledge-based acquisition of rules for medical diagnosis, Journal of medical systems, Vol. 6, pp. 433-445, 1982
[11] R. Bock, J. Meier, L. G. Nyúl, et al. Glaucoma risk index: Automated glaucoma detection from color fundus images, Medica image analysis, Vol. 14, pp. 471-481, 2010

[12] J. Liu, Z. Zhang, D. W. K. Wong, et al. Automatic glaucoma diagnosis through medical imaging informatics, Journal of the American Medical Informatics Association, Vol. 20, pp. 1021-1027, 2013.

[13] J. Nayak, R. Acharya, P. S. Bhat, et al. Automated diagnosis of glaucoma using digital fundus images, Journal of medical systems, Vol. 33, pp. 337-346, 2009.

[14] J. Cheng, J. Liu, Y. Xu, et al. Superpixel Classification based Optic Disc and Optic Cup Segmentation for Glaucoma Screening, IEEE Transactions on Medical Imaging, Vol. 32, pp. 1019 - 1032, 2013.

[15] Z. Zhang, C.K. Kwoh, J. Liu, et al. MRMR optimized classification for automatic glaucoma diagnosis, Engineering in Medicine and Biology Society, EMBC, 2011 Annual International Conference of the IEEE, pp. 6228-6231, 2011.

[16] C. Cortes, V. N. Vapnik, Support vector networks, Machine learning, Vol. 20, pp. 273-297, 1995.

[17] D.R. Chen, Q. Wu, Y. Ying, D.X. Zhou, Support vector machine soft margin classifiers: error analysis, The Journal of Machine Learning Research, Vol. 5, pp. 1143-1175, 2004

[18] H. L. Chen, B. Yang, G. Wang, et al., Support vector machine based diagnostic system for breast cancer using swarm intelligence, Journal of medical systems, Vol. 36, pp. 2505-2519, 2012.

[19] A. Ozcift, SVM feature selection based rotation forest ensemble classifiers to improve computer-aided diagnosis of Parkinson disease, Journal of medical systems, Vol. 36, pp. 2141-2147, 2012

[20] G. D. Joshi, J. Sivaswamy, S. R. Krishnadas, Optic Disk and Cup Segmentation from Monocular Color Retinal Images for Glaucoma Assessment, IEEE Transactions on Medical Imaging, Vol. 30, pp. 1192-1205, 2011

[21] I. T. Jolliffe, Principal Component Analysis, Series: Springer Series in Statistics, 2nd ed., Springer, NY, 2002. 